\title{
Stimulation-Induced Within-Representation and Across-Representation Plasticity in Human Motor Cortex
}

\author{
Ulf Ziemann, George F. Wittenberg, and Leonardo G. Cohen \\ Human Cortical Physiology Section, National Institute of Neurological Disorders and Stroke, National Institutes of Health, \\ Bethesda, Maryland 20892-1428
}

\begin{abstract}
The human motor cortex contains a dynamic and distributed network of motor representations. Formation, maintenance, and modification of these representations is an activity-driven process. Repeated stimulation of one representation results in increased motor output from this representation, a process referred to as "within-representation plasticity." We showed previously that within-representation plasticity of the upper arm representation occurs when repetitive transcranial magnetic stimulation (rTMS) is delivered to that representation during transient ischemic nerve block (INB) of the contralateral hand. INB reduces inhibition in the motor cortex and thus lowers the threshold for stimulation-induced plasticity. Here we studied the effects of rTMS delivered to nearby body part representations on the motor output from the upper arm representation. Six healthy subjects underwent INB-alone (control), or INB plus $30 \mathrm{~min}$ of focal $0.1 \mathrm{~Hz}$ rTMS of either the face, hand, arm, leg, or overlap arm/hand representations in motor cortex. INB-alone
\end{abstract}

There is a general somatotopic order of representations in primary motor cortex, but representations may overlap, as with arm and hand representations (Penfield and Boldrey, 1937; Wassermann et al., 1992; Rao et al., 1995). This arm/hand area can be viewed as a network of distributed and overlapping multiple motor representations that are connected extensively through long-range horizontal fibers. This network is dynamic and maintains the capacity for plasticity throughout life (Sanes and Donoghue, 2000).

One experimental form of plasticity is stimulation-induced plasticity. Seminal experiments were undertaken at the beginning of the last century and found that repeated stimulation of one point in monkey motor cortex produced marked facilitation of motor output from this point (Grünbaum and Sherrington, 1903; Graham Brown and Sherrington, 1912). More recent experiments in adult rat motor cortex showed that repetitive intracortical microstimulation resulted in a sustained $(\sim 30 \mathrm{~min})$ expansion of the stimulated motor representation (Nudo et al., 1990). Analogous experiments in rat and monkey primary somatosensory cortex resulted in expansion of the stimulated cortical skin representation (Recanzone et al., 1992).

Received Sept. 21, 2001; revised April 15, 2002; accepted April 25, 2002.

This work was supported by Grant ZI 542/1-1 (U.Z.) from the Deutsche Forschungsgemeinschaft.

Correspondence should be addressed to Dr. Ulf Ziemann, Clinic of Neurology, JW Goethe University of Frankfurt, Theodor-Stern-Kai 7, D-60590 Frankfurt am Main, Germany. E-mail: u.ziemann@em.uni-frankfurt.de. or to Dr. Leonardo G. Cohen, Human Cortical Physiology Section, NINDS, NIH, Building 10, Room SN242, 10 Center Drive, Bethesda, MD 20892-1430. E-mail: cohenl@ninds.nih.gov.

U. Ziemann's present address: Clinic of Neurology, JW Goethe University of Frankfurt, D-60590 Frankfurt am Main, Germany.

Copyright (C) 2002 Society for Neuroscience $\quad 0270-6474 / 02 / 225563-09 \$ 15.00 / 0$ and rTMS of the leg representation resulted in only a shortlasting $(<20 \mathrm{~min})$ increase in motor output from the arm representation, as measured by motor evoked potentials in the biceps. rTMS of arm and arm/hand representations induced a prolonged ( $>60 \mathrm{~min}$ ) within-representation increase. In contrast, rTMS of face or hand representations canceled the shortlasting increase and even led to a long-lasting decrease of motor output from the arm representation. Therefore, rTMS of the experimentally disinhibited motor cortex induces withinrepresentation increase, and across-representation decrease of motor cortical output. This bidirectional plasticity might be used for purposeful modulation of human cortical function.

Key words: stimulation-induced plasticity; input-specificity; across-representation plasticity; transient ischemic nerve block; transcranial magnetic stimulation; repetitive stimulation; motor evoked potential; human motor cortex

Much less is known about stimulation-induced plasticity across representations. In motor cortex, there are potential interactions between antagonistic motor representations within the same body part representation (e.g., elbow flexion vs elbow extension), or between different body representations (e.g., face vs arm). Acrossrepresentation plasticity may play an important role in the recovery of function in animals with lesions (Sanes et al., 1988; Donoghue et al., 1990; Nudo and Milliken, 1996; Nudo et al., 1996); for review, (Kaas, 1991) and in patients with neurological disorders (Rijntjes et al., 1997; Liepert et al., 1999; Karl et al., 2001). Furthermore, across-representation plasticity may reveal connections between different body part representations, which in motor cortex are likely to be inhibitory (Kujirai et al., 1993; Baker et al., 1998).

To our knowledge, only early reports (Graham Brown and Sherrington, 1912) addressed the issue of across-representation plasticity experimentally. Repetitive stimulation of the elbow flexion representation in monkey motor cortex usually resulted in inhibition of motor output from the antagonistic motor representation of elbow extension, and vice versa (Graham Brown and Sherrington, 1912).

We demonstrated previously the existence and properties of stimulation-induced within-representation plasticity in human motor cortex, experimentally disinhibited by ischemic nerve block (INB) (Brasil-Neto et al., 1992; Ziemann et al., 1998a,b). Repetitive transcranial magnetic stimulation (rTMS) of the upper arm representation resulted in a long-lasting increase in motor output from that representation.

In the present study, we tested the hypothesis that, in the 
Experimental Manipulation
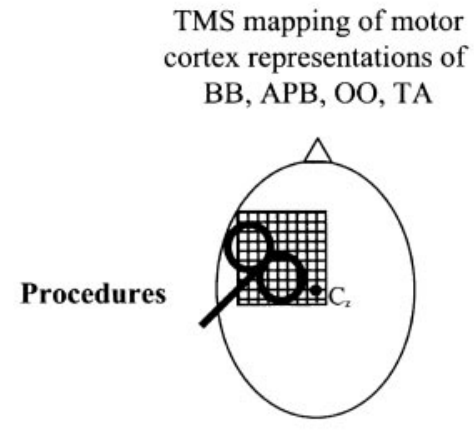

COG referenced

Measures to $\mathrm{Cz}$ for $\mathrm{BB}$, APB, OO, TA

Days to weeks prior to the manipulation experiments

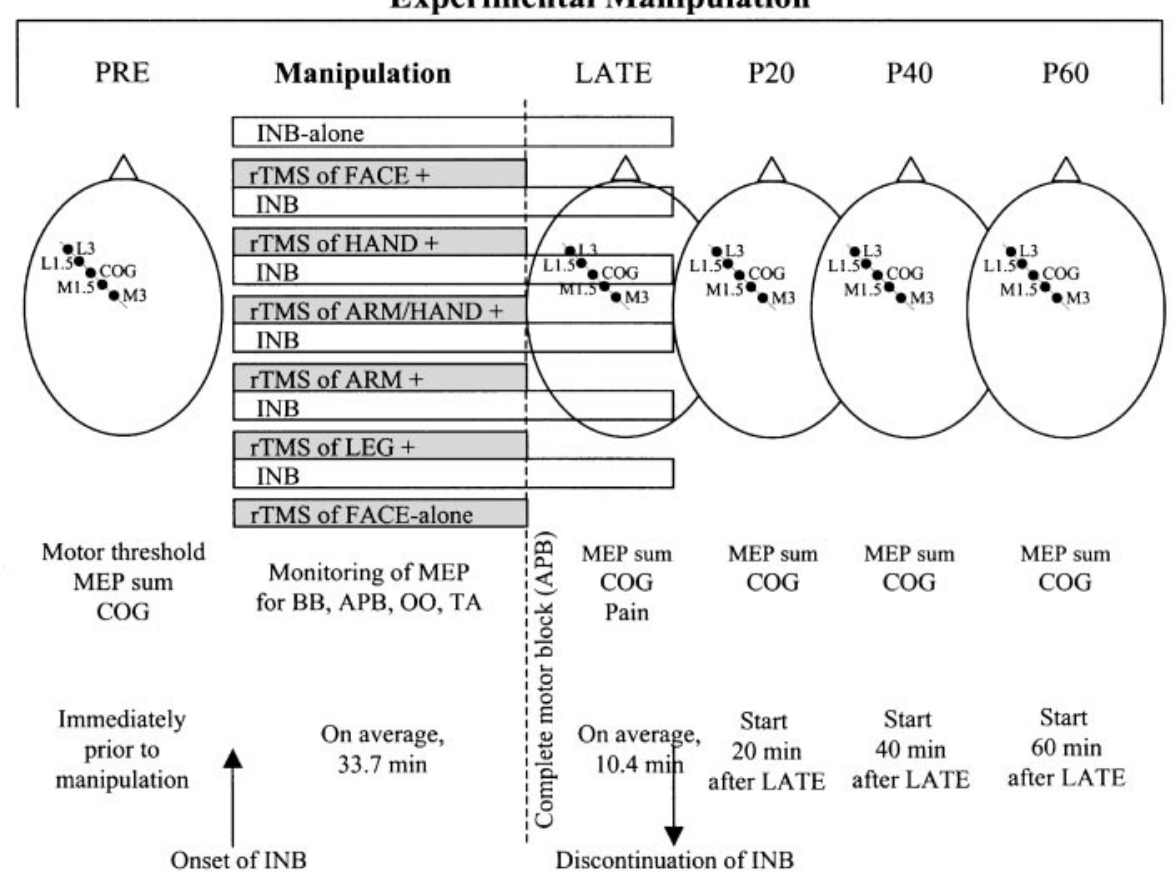

Figure 1. Time line of experimental procedures. First, detailed two-dimensional maps in motor cortex of the BB, APB, OO, and TA were obtained, using an eight-shaped coil that was moved along a $1 \times 1 \mathrm{~cm}$ grid referenced to the vertex $\left(C_{z}\right.$ left-most panel; subject's head viewed from above). Days later, seven different manipulation experiments separated by at least 1 week were conducted in randomized order. Immediately before each manipulation (PRE), excitability (motor threshold, MEP sum) and location $(C O G)$ of the $\mathrm{BB}$ map were measured along one axis, testing five grid points $1.5 \mathrm{~cm}$ apart ( panel beneath PRE). The central point $(C O G)$ corresponded to the BB COG obtained in the initial two-dimensional mapping experiment. The manipulations consisted either of ischemic nerve block of the hand (the INB-alone control experiment), focal $0.1 \mathrm{~Hz}$ rTMS of the face representation in motor cortex (the FACE-alone control experiment), or the combination of rTMS delivered to one of five different sites (FACE, HAND, ARM, LEG, or ARM/HAND overlap representation in motor cortex) and INB. The onset of INB (inflation of a tourniquet at the forearm) is indicated by the upward facing arrow. MEP in the BB, APB, OO, and TA elicited by rTMS were monitored throughout. Complete motor block to the APB was reached on average after $33.7 \mathrm{~min}$ (indicated by the vertical dashed line). At this point (in the FACE-alone experiment after 30 min) rTMS was stopped, and the LATE measurements of the BB map were started following the same protocol as the PRE measurements ( panel beneath LATE). Immediately thereafter INB was discontinued (downward facing arrow). Further measurements of the BB map were obtained 20, 40, and 60 min $(P 20, P 40, P 60)$ after the end of the LATE measurements. The data were analyzed by comparing the LATE, P20, P40, and P60 measures with the PRE measures, using two-way repeated measure ANOVA with experimental manipulation and time as the within-subject effects.

experimentally disinhibited motor cortex, rTMS of other body part representations (face, hand, leg) would result in an acrossrepresentation decrease of motor output from the arm representation. If so, this would support the idea that interactions between different body part representations in human motor cortex are generally inhibitory. Furthermore, such opposite effects of stimulation-induced within-representation increase versus acrossrepresentation decrease of motor cortical output might open a window for purposeful modulation of human cortical function.

\section{MATERIALS AND METHODS}

Subjects. Six healthy subjects (five male, four right-handed; mean age, $35 \pm 11$ years) participated in a detailed mapping experiment, and subsequently, in seven different manipulation experiments. All subjects gave their written informed consent for the study. The study protocol was approved by the National Institute of Neurological Disorders and Stroke Institutional Review Board.

Two-dimensional motor cortex mapping. The time line of experiments is shown in Figure 1. In all experiments, subjects were seated in a comfortable reclining chair. For each subject, the first experiment consisted of a detailed two-dimensional mapping of four body part representations (arm, hand, face, leg) in motor cortex to determine the exact stimulation sites for the subsequent manipulation experiments (see below). The four body part representations were mapped by recording motor-evoked potentials (MEPs) from one target muscle from each representation. The target muscles were the biceps brachii (BB) and abductor pollicis brevis (APB) muscles of the nondominant arm and hand, and the orbicularis oculi (OO) and tibialis anterior (TA) muscles of the face and leg of the same side. Bipolar surface electromyography (EMG) was recorded from these muscles, using tin disk electrodes and a four-channel Dantec Electronics (Skovlunde, Denmark) Counterpoint Electromyograph. The EMG was amplified, bandpass filtered (0.1-2.5 $\mathrm{kHz}$ ), and stored (analog-to-digital rate, $5 \mathrm{kHz}$ ) on an IBM/486 ATcompatible laboratory computer for off-line analysis. Focal TMS was delivered through an eight-shaped (diameter of each wing, $70 \mathrm{~mm}$ ) stimulating coil (peak magnetic field, $2.2 \mathrm{~T}$ ) connected to a Magstim (Whitland, Dyfed, UK) 200 magnetic stimulator. The coil was placed tangentially to the scalp over the nondominant motor cortex, and the handle pointed backward and $\sim 45^{\circ}$ away from the midline. Thus, the current induced in the brain ran from posterolateral to anteromedial, approximately perpendicular to the central sulcus. This ensured a predominantly trans-synaptic activation of the corticospinal system (Kaneko et al., 1996). For the mapping, a $1 \times 1 \mathrm{~cm}$ grid was drawn on the scalp over the presumed area of the motor cortex by a fiber-tipped pen. The coordinates of this grid were referenced to the vertex (Fig. 1, left panel, $C_{z}$ ). A provisional optimal coil position was determined as the site that resulted consistently in maximal MEP of the actual target muscle (subsequently $\mathrm{BB}, \mathrm{APB}, \mathrm{OO}, \mathrm{TA}$ ) at a moderately suprathreshold stimulus intensity (Classen et al., 1998). At this position, the resting motor threshold was determined to the nearest $1 \%$ of maximum stimulator output. Resting motor threshold was defined as the minimum stimulus intensity that produced MEP $>50 \mu \mathrm{V}$ in at least 5 of 10 consecutive trials (Rossini et al., 1994). A detailed mapping was then performed at a stimulus intensity of $120 \%$ motor threshold. The mapping was started at the provisional optimal position and continued at increasingly more peripheral points of the grid. At each site, five trials were performed. The mapping was complete when "positive" sites (MEP $>50 \mu \mathrm{V}$ in at least two of five trials) were entirely surrounded by "negative" sites (coil 
positions in which TMS did not elicit MEP $>50 \mu \mathrm{V}$ in at least two of five trials). The single trial peak-to-peak MEP amplitudes were analyzed, and the center of gravity (COG) was calculated using the formula $\Sigma($ MEP $\times$ site $) / \Sigma M E P$ (i.e., the sum of MEP weighted individually by vector of site position divided by the sum of all MEP). The average BB COG coordinates were $4.6 \pm 0.9 \mathrm{~cm}$ lateral and $0.6 \pm 0.8 \mathrm{~cm}$ anterior to $\mathrm{C}_{7}$. The maps of BB and APB showed extensive overlap with the APB COG slightly more lateral $\left(5.1 \pm 0.7 \mathrm{~cm}\right.$ lateral to $\left.\mathrm{C}_{z}\right)$ and anterior $(0.9 \pm$ $0.6 \mathrm{~cm}$ anterior to $\mathrm{C}_{\mathrm{z}}$ ) compared with the $\mathrm{BB} \mathrm{COG}$. The coordinates of the OO COG were $8.9 \pm 1.4 \mathrm{~cm}$ lateral and $2.1 \pm 1.1 \mathrm{~cm}$ anterior to $\mathrm{C}_{\mathrm{z}}$, and those of the TA COG $1.3 \pm 0.7 \mathrm{~cm}$ lateral and $0.4 \pm 0.6 \mathrm{~cm}$ posterior to $\mathrm{C}_{\mathrm{z}}$. The Euclidean distances between the BB COG and the COG of the APB, OO, and TA were $0.6 \pm 0.3,5.0 \pm 0.3$, and $3.7 \pm 0.7 \mathrm{~cm}$, respectively. Coordinates and distances are consistent with previous TMS (Wassermann et al., 1992; Classen et al., 1998) and movementrelated potential (Cheyne et al., 1991) mapping studies of human motor cortex. The two-dimensional mapping of the four body part representations in motor cortex was done in each subject several days to weeks before the manipulation experiments.

Excitability and location of the arm representation immediately before experimental manipulation. The aim of this study was to test changes in the arm representation in motor cortex produced by different experimental manipulations. As a baseline, excitability and location of the arm representation were measured [before experimental manipulation (PRE) measurements] by one-dimensional mapping of BB MEP immediately before each experimental manipulation (see below). To this end, the individual BB COG, as determined in the initial two-dimensional mapping (see above) and two medial (M1.5, M3) and two lateral sites (L1.5, L3) were marked with a pen on the scalp (Fig. 1, panel below PRE). Adjacent positions were separated by $1.5 \mathrm{~cm}$ and were placed along an axis from anterior-lateral to posterior-medial, i.e., approximately parallel to the central sulcus. Excitability and location of the arm representation were then measured as follows: BB resting motor threshold at each of the five sites, following the protocol for determination of motor threshold given above. At each site, MEP were then obtained with one block of trials at motor threshold intensity as determined for each site, and with two further blocks at intensities 15 and $30 \%$ of maximum stimulator output above motor threshold. Five trials were delivered at each site and for each intensity (i.e., five trials $\times$ five sites $\times$ three intensities $=75$ trials). The intertrial interval was $5 \mathrm{sec}$. Various measures were extracted from these mapping data. The peak-to-peak amplitude of the MEP was analyzed in the single trials. Then MEP amplitudes were averaged for each stimulation site and stimulation intensity. The MEP sum was defined as the sum of MEP peak-to-peak amplitudes across all stimulation sites and across the two suprathreshold stimulation intensities (15 and 30\% above motor threshold). The COG was derived from this set of data as the MEP amplitude weighted by stimulation site and divided by the MEP sum: $\mathrm{COG}=\Sigma($ MEP $\times$ site $) / \mathrm{MEP}$ sum. Therefore, the PRE measures consisted of motor threshold, MEP sum, and $\mathrm{COG}$ of the BB.

Experimental manipulations. Immediately after determination of the PRE measures, one of seven different experimental manipulations was performed (Fig. 1, manipulation). Each subject was tested with each of the manipulations (six subjects $\times$ seven manipulations $=42$ experiments). The order of manipulations across subjects was pseudorandomized and balanced. The control manipulation experiment consisted of ischemic nerve block (INB) alone. A tourniquet was inflated at the level of the proximal forearm, distal to the BB muscle, to $220-250 \mathrm{mmHg}$, i.e., well above systolic blood pressure. The pressure was kept constant until complete motor block was achieved and the late into INB excitability measurements (LATE, see below) were completed (total INB time, on average, $44.3 \pm 5.0 \mathrm{~min}$ ) (Fig. 1 ). Thereafter, the tourniquet was quickly released. Complete motor block was defined as the time when MEP were no longer elicited in the APB in three consecutive trials at $30 \%$ above motor threshold stimulation intensity (on average, $33.9 \pm 4.6 \mathrm{~min}$ ).

In five manipulation experiments, INB was combined with low-rate $(0.1 \mathrm{~Hz})$ focal rTMS delivered to one of five different sites (FACE, HAND, ARM/HAND overlap area, ARM, and LEG). rTMS always started with tourniquet inflation and stopped when complete motor block was achieved (Fig. 1, gray horizontal bars below manipulation). Throughout the period of rTMS, the EMG from all four target muscles (BB, APB, $\mathrm{OO}$, and TA) was recorded to monitor the distribution of MEP elicited by rTMS (Fig. 2). This was rather important to demonstrate the topographic specificity of the rTMS effects in the different experimental manipulations. In the ARM/HAND+INB condition, rTMS was deliv-
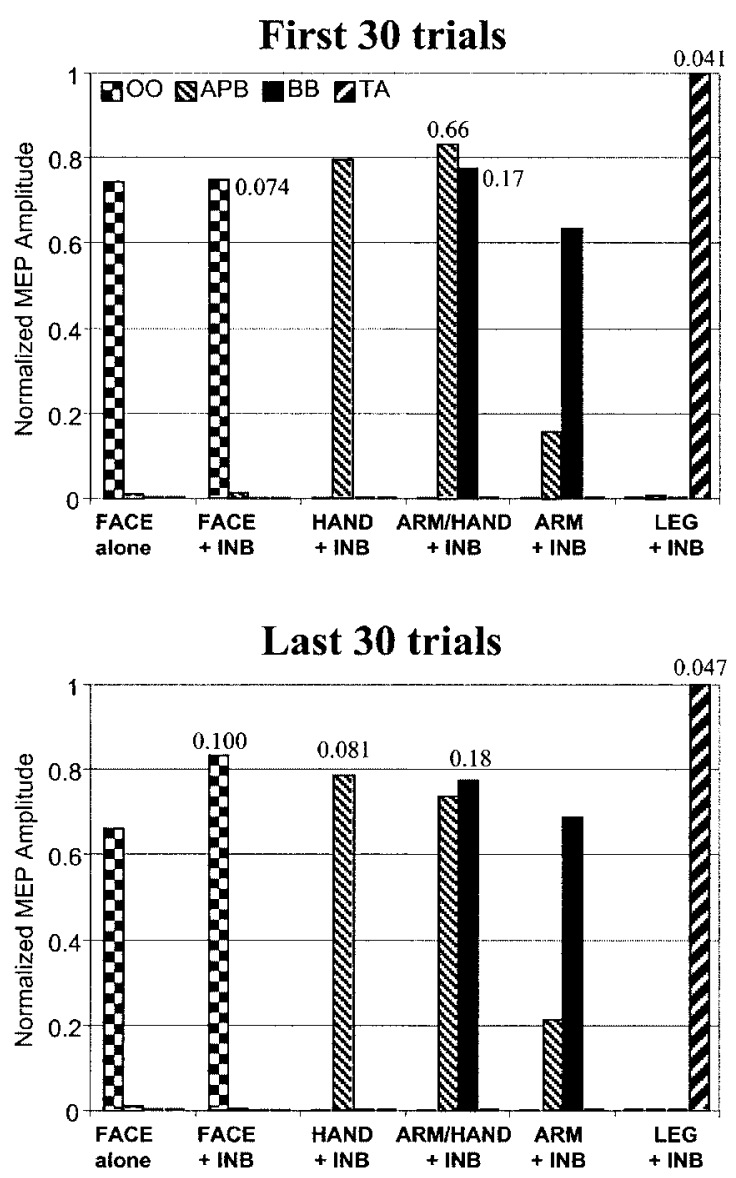

Figure 2. Normalized amplitudes of the MEP of the four different target muscles (OO, APB, BB, and TA), as elicited during experimental manipulation by repetitive transcranial magnetic stimulation delivered to different sites of the motor cortex (FACE, HAND, ARM/HAND overlap area, ARM, LEG; for definition, see Materials and Methods). INB, Ischemic nerve block of the hand. The top panel refers to the first 30 trials after start of INB ( $5 \mathrm{~min}$ ), and the bottom panel to the last 30 trials before reaching complete motor block in the APB. Length of bars ( $y$-axis) reflects the MEP amplitude normalized to the maximum amplitude for a given target muscle and subject $(n=6)$ across stimulation sites. Numbers indicate the mean maximum MEP amplitudes of the different target muscles (in millivolts).

ered to the BB COG with the intensity of rTMS set to $120 \%$ of BB motor threshold. These rTMS parameters were shown previously not to produce any significant change in MEP amplitude in the absence of INB (Chen et al., 1997; Ziemann et al., 1998a). In this condition, consistently in all subjects, rTMS elicited MEP in the BB and APB because of the extensive overlap of the two representations (Fig. 2). In the HAND + INB condition, rTMS was delivered to the L3 site at an intensity just below BB motor threshold at this site, resulting in APB MEP only, but not BB MEP (Fig. 2). In the ARM + INB condition, rTMS was pointed to the M3 site at an intensity just below APB motor threshold, resulting in MEP predominantly in the BB, but usually not in the APB (Fig. 2). In the FACE + INB and LEG + INB conditions, rTMS was delivered to the $\mathrm{OO} \mathrm{COG}$ and $\mathrm{TA} \mathrm{COG}$, respectively, as obtained in the initial two-dimensional mapping (see above). rTMS intensities were set to $120 \%$ of motor threshold in the OO or TA. This resulted in isolated MEP of either the OO or TA, without MEP in the APB or BB (Fig. 2). Finally, in one more control manipulation experiment, rTMS was delivered to the face representation in the absence of INB (FACE-alone condition). In this experiment, the duration of rTMS was set to $30 \mathrm{~min}$. The delay between consecutive experiments in a given subject was at least 1 week.

To test the effects of experimental manipulation, excitability and location of the arm representation were measured the same way as the PRE measurements right at the end of each of the different experimental 
Table 1. Excitability and location of the arm representation in motor cortex before manipulation

\begin{tabular}{|c|c|c|c|c|c|c|c|c|c|}
\hline & INB & $\mathrm{LEG}+\mathrm{INB}$ & $\mathrm{ARM}+\mathrm{INB}$ & $\begin{array}{l}\text { ARM/HAND } \\
+ \text { INB }\end{array}$ & HAND + INB & $\mathrm{FACE}+\mathrm{INB}$ & FACE alone & $F$ & $p$ \\
\hline $\mathrm{MT}_{\mathrm{COG}}$ & 39.7 & 37.7 & 38.8 & 37.7 & 39.2 & 38.2 & 39.6 & 0.54 & NS \\
\hline $\mathrm{MT}_{\mathrm{L} 1.5}$ & 46.8 & 46.0 & 44.3 & 41.3 & 44.2 & 43.7 & 47.4 & 1.37 & NS \\
\hline $\mathrm{MT}_{\mathrm{L} 3}$ & 63.3 & 66.7 & 61.8 & 58.1 & 59.5 & 63.3 & 62.4 & 2.12 & NS \\
\hline $\mathrm{MT}_{\mathrm{M} 1.5}$ & 46.3 & 43.5 & 43.5 & 45.1 & 45.8 & 43.3 & 46.4 & 0.79 & NS \\
\hline $\mathrm{MT}_{\mathrm{M} 3}$ & 63.0 & 64.0 & 59.7 & 62.1 & 65.8 & 64.7 & 67.4 & 0.87 & NS \\
\hline MEP sum & 2.0 & 2.6 & 2.0 & 2.9 & 3.6 & 3.1 & 3.5 & 1.13 & NS \\
\hline$\Delta \mathrm{COG}$ & 0.060 & 0.065 & 0.077 & -0.014 & -0.105 & -0.036 & -0.017 & 0.16 & NS \\
\hline
\end{tabular}

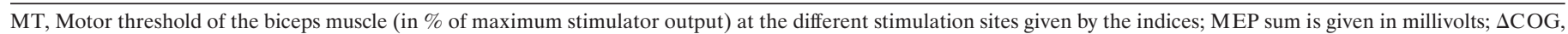
deviation from COG (in centimeters, negative values indicate deviation lateral from COG). All values are means of six subjects.

Table 2. Time to complete motor block, total duration of INB, and level of INB-induced pain

\begin{tabular}{|c|c|c|c|c|c|c|c|c|}
\hline & INB & $\mathrm{LEG}+\mathrm{INB}$ & $\mathrm{ARM}+\mathrm{INB}$ & $\begin{array}{l}\text { ARM/HAND } \\
+ \text { INB }\end{array}$ & HAND + INB & FACE + INB & $F$ & $p$ \\
\hline CMB & 33.3 & 34.8 & 34.2 & 31.7 & 33.1 & 35.0 & 1.65 & NS \\
\hline tINB & 42.3 & 44.7 & 44.2 & 42.1 & 43.6 & 47.5 & 4.30 & 0.006 \\
\hline Pain & 10.2 & 9.7 & 10.7 & 9.3 & 8.8 & 11.2 & 0.96 & NS \\
\hline
\end{tabular}

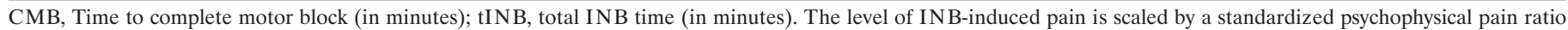
scale (maximum 20). All values are means of six subjects.

manipulations (Fig. 1, LATE measurements) and 20, 40, and 60 min later (Fig. 1, P20, P40, and P60 measurements). Single-pulse TMS used for these measurements was equally presented in all conditions and therefore cannot account for the differences observed between experimental manipulations (see below). In addition, in all INB experiments, the intensity of the INB-induced pain was evaluated immediately before tourniquet release by means of a standardized psychophysical pain ratio scale (0-20 points) (Max et al., 1992) with good internal consistency, reliability, and objectivity (Gracely et al., 1978).

Excitability and location of the face representation. An additional limited set of measurements was performed in the OO muscle, in addition to the measurements in the $\mathrm{BB}$, because we were interested in the relation between possible manipulation-induced changes of the face and arm representations. In four of the six subjects, motor threshold, MEP sum, and $\mathrm{COG}$ of the $\mathrm{OO}$ muscle were determined before and in the course of the FACE + INB and ARM + INB experiments, in addition to the measures in the BB (see above). For the one-dimensional OO mapping, the stimulation points were extended by two sites 4.5 and $6 \mathrm{~cm}$ lateral to COG (L4.5, L6). The OO mapping was limited to one block of trials, using stimulus intensities equal to BB motor threshold at the BB COG, L1.5, and L3 sites. The stimulation intensity at the L4.5 and L6 sites was set to the one at the L3 site. This, on average, was equal to $16.8 \pm 5.5 \%$ of the maximum stimulator output above the motor threshold of the $\mathrm{OO}$ muscle (determined at the optimal position for eliciting MEP in OO, either at L4.5 or at L6). Higher stimulation intensities were not tested because most subjects did not tolerate high-intensity TMS over the temporalis muscle because of the painful muscle twitch induced.

Statistics. We sought to exclude significant effects of the individual experiment PRE measures (motor threshold, MEP sum, COG), the time to reach complete motor block, the total duration of INB, and the level of INB-induced pain on the results produced by the different experimental manipulations. Therefore, these measures were evaluated by separate repeated-measures one-way ANOVA with experimental manipulation as the within-subject effect.

To evaluate the effects of experimental manipulation on MEP sum and COG, the postmanipulation measures (LATE, P20, P40, P60) were related to the PRE measures: (post - pre)/pre (MEP sum), or post - pre $(\mathrm{COG})$. These values were then evaluated separately by repeatedmeasures two-way ANOVA with experimental manipulation and time as the within-subject effects. Accordingly, the effects of experimental manipulation measured in parallel for $\mathrm{BB}$ and $\mathrm{OO}$ were analyzed with repeated-measures three-way ANOVA with muscle, experimental manipulation, and time as the within-subject effects. Conditional on a significant $F$ value, post hoc analyses were performed using paired Student's $t$ tests, adjusted for multiple comparisons by the Bonferroni method. Differences were deemed significant if $p<0.05$.

\section{RESULTS}

\section{Excitability and location of the arm representation in motor cortex before manipulation}

Motor threshold, MEP sum, and COG of the BB immediately before experimental manipulation (PRE measures) were not different between the various manipulations (Table 1). Therefore, the differential effects of experimental manipulation on excitability and location of the arm representation (see below) were not caused by differences in initial state.

\section{Time to complete motor block, total duration of INB, and level of INB-induced pain}

The time to reach complete motor block and the level of INBinduced pain were not different between experimental manipulations (Table 2). The total duration of INB was significantly different across manipulations (Table 2). Post hoc comparisons revealed that the duration of INB was slightly longer (by $<4 \mathrm{~min}$ ) in the FACE + INB than in the INB-alone condition $(p=$ $0.0005)$ and in the FACE + INB than in the ARM/HAND + INB condition $(p=0.0012)$. These differences were accounted for by the slightly longer duration of the LATE measurements in the FACE + INB condition because of the addition of MEP mapping of the $\mathrm{OO}$ muscle in this condition. However, because the most important contrasting effects of the different manipulations did not rely on the FACE + INB condition (see below), it can be safely assumed that differences in time to reach complete motor block, total INB duration, and the level of INB-induced pain did not account for the differential effects of experimental manipulation on excitability and location of the arm representation (see below).

\section{MEP elicited with rTMS}

During rTMS (Fig. 1, gray bars), monitoring of all four muscles (BB, $\mathrm{APB}, \mathrm{OO}$, and TA) revealed that $\mathrm{BB}$ MEP were elicited with the ARM + INB and ARM/HAND + INB (overlap area) conditions only (Fig. 2, black bars). APB MEP were elicited with the HAND + INB and ARM/HAND + INB conditions only, and to a much lesser extent and only inconsistently with the 
FACE+INB

HAND+INB

PRE

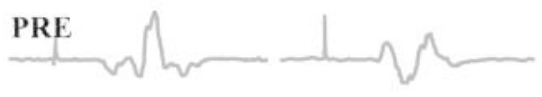

LATE

P20 M $M$

$\underbrace{P 60}$
ARM/HAND+INB

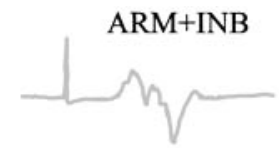

(

$\rightarrow \ln 4+44 \%$

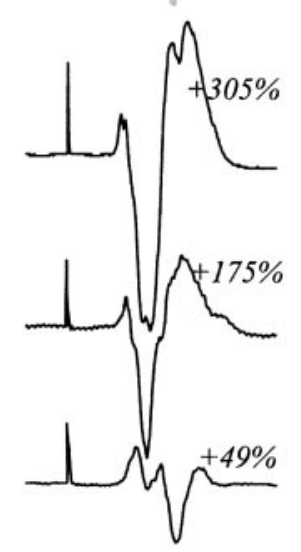

INB-alone
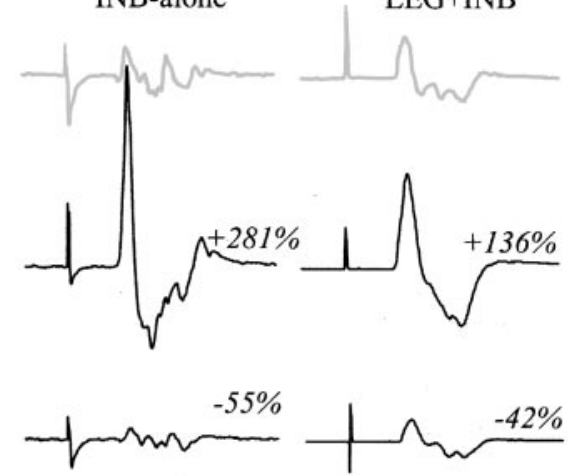

-

$20 \mathrm{~ms}$

Figure 3. Changes in MEP amplitude in the biceps muscle of one representative subject induced by six different experimental manipulations (FACE, HAND, ARM/HAND, ARM, LEG $=0.1 \mathrm{~Hz}$ focal rTMS of the face, hand, arm/hand overlap, upper arm and leg representations in motor cortex, respectively, combined with INB). All recordings are averages of five trials obtained at the center of gravity of the biceps muscle using stimulation intensity of $30 \%$ above biceps motor threshold. Gray curves (top row) indicate MEP before manipulation (PRE), the other curves show MEP late into INB (LATE), and 20 and 60 min after the end of INB (P20, P60). Percentage values indicate the change of MEP amplitude compared with PRE. Note the long-lasting MEP increase with ARM/HAND + INB and ARM + INB compared with the only transient increase with INB-alone. Note further the much weaker transient increase with HAND + INB and FACE + INB compared with INB-alone.

ARM + INB condition (Fig. 2, finely graded cross-hatched bars). In the TA and OO, MEP were elicited only with the LEG + INB condition and with the FACE-alone and FACE + INB conditions, respectively (Fig. 2). There were no significant differences in the distribution of MEP elicited by rTMS at the start of INB (Fig. 2, top panel) and immediately before complete motor block was reached (Fig. 2, bottom panel). These data indicate that selective stimulation of the various motor representations was successfully achieved throughout the different experimental manipulations. This is a rather important result, suggesting that the differential effects of manipulation on excitability and location of the arm representation (see below) were determined by specific stimulation of a motor representation by rTMS.

\section{Effects of experimental manipulation on MEP sum of the arm representation.}

The principal findings of the present study are shown in Figure 3 (original BB MEP recordings from one representative subject) and Figure 4 (BB MEP sum group data). In the single subject, BB MEP amplitude greatly increased $(>200 \%)$ at the end of experimental manipulation (LATE) in the ARM/HAND + INB, $\mathrm{ARM}+\mathrm{INB}$, and INB-alone conditions, whereas much lesser increases occurred in the FACE + INB and HAND + INB conditions (Fig. 3). Furthermore, the BB MEP increase was sustained over $60 \mathrm{~min}$ in the ARM/HAND + INB and ARM + INB conditions, compared with the transient effect in the INBalone condition (Fig. 3).

ANOVA of the MEP sum group data (Fig. 4) revealed significant effects of manipulation $(F=4.66 ; p=0.0018)$ and time $(F=$ 4.42; $p=0.02$ ). Post hoc comparisons showed a significantly longer-lasting increase in BB MEP sum in the ARM/HAND + INB and ARM + INB conditions compared with the only transient increase in the INB-alone condition (significance levels of the post hoc paired $t$ tests are given in Fig. 4). These data

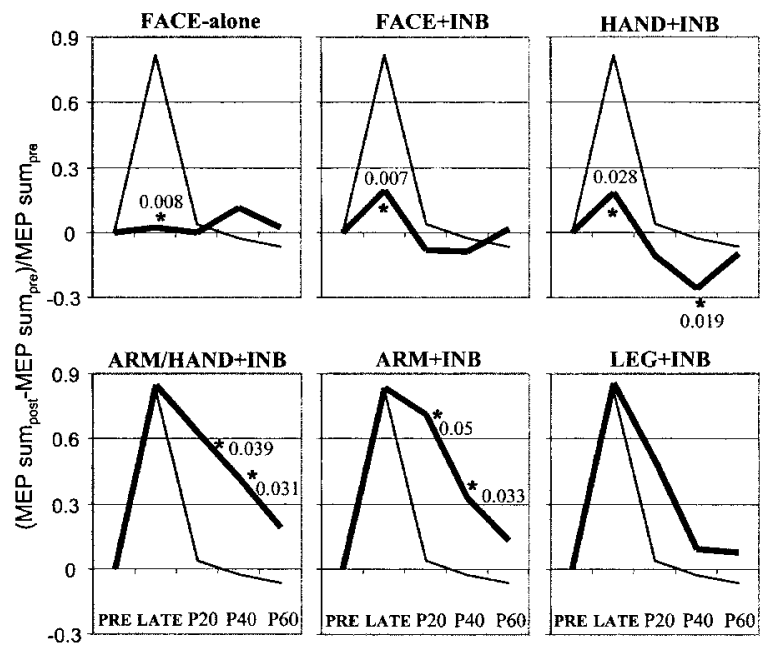

Figure 4. Changes in MEP sum of the biceps muscle (means of six subjects) over time. The thin curves in all diagrams refer to the control experiment (INB-alone). The thick curves refer to the experimental manipulation indicated at the top of each diagram (for abbreviations, see Materials and Methods). The time of MEP sum measurements is given on the $x$-axis (for abbreviations, see Fig. 1). Asterisks indicate significant differences between the two curves in a given diagram. $p$ values (paired two-tailed $t$ tests) are also shown. Note that INB-alone resulted in only a transient increase of MEP sum, whereas ARM/HAND + INB and $\mathrm{ARM}+\mathrm{INB}$ led to a long-lasting increase. In contrast, FACE + INB and HAND + INB canceled the transient increase obtained with INBalone, and HAND + INB even resulted in a long-lasting decrease of MEP sum. LEG + INB was not significantly different from INB-alone.

replicate previous findings (Ziemann et al., 1998a). They suggest that stimulation of one representation in motor cortex (in this case the arm representation) during release from cortical inhibition (in this case induced by INB) results in a long-lasting 
within-representation increase of motor output from the stimulated representation.

In contrast, the short-lasting increase in BB MEP sum was significantly reduced in the HAND + INB and FACE + INB conditions compared with the INB-alone condition (significance levels of the post hoc paired $t$ tests are given in Fig. 4). Furthermore, HAND + INB resulted in a long-lasting ( $\sim 40 \mathrm{~min})$ significant decrease in BB MEP sum compared with the INB-alone condition (Fig. 4). These data suggest that stimulation of representations adjacent to the arm representation (hand, face) during release from cortical inhibition results in a long-lasting decrease across-representations of motor output of the nonstimulated arm representation. This supports the notion of a primarily inhibitory interaction of the face and hand representations with the arm representation, in the experimentally disinhibited human motor cortex.

LEG + INB resulted in an increase of BB MEP sum that was similar to the one obtained with INB-alone. Although the data suggest a more sustained increase with LEG + INB than with INB-alone (Fig. 4), the difference was not significant. The lack of a significant difference between the LEG + INB and INB-alone conditions may be explained by the idea that there exist no relevant interaction between the leg and arm representations or by the fact that the leg representation is not adjacent to the deprived hand cortex.

Finally, FACE-alone (in the absence of INB) did not result in changes of BB MEP sum (Fig. 4). This indicates that an inhibitory effect of FACE + INB compared with INB-alone was measurable only during a condition of reduced cortical inhibition.

\section{Effects of experimental manipulation on COG of the arm representation.}

Although ANOVA did not show any significant effects of manipulation $(F=1.44 ; p=0.24)$ or time $(F=0.95 ; p=0.44)$ on the BB COG (Fig. 5A,B), there was a slight mean medial shift of the BB COG by $0.2 \mathrm{~cm}$ with the INB-alone condition. In contrast, the ARM/HAND + INB manipulation, and more conspicuously the HAND + INB manipulation, resulted in a mean lateral shift of the $\mathrm{BB} C \mathrm{COG}$ by up to $0.3 \mathrm{~cm}$, gradually regressing back to the original position over time (Fig. $5 A, B$ ). The BB COG shifts in the FACE + INB and LEG + INB conditions were minimal and not significantly different from the INB-alone condition.

\section{Effects of experimental manipulation on MEP sum and COG of the face representation}

Figure $6 A$ illustrates that, for one representative subject, FACE + INB resulted in a long-lasting increase $(>60 \mathrm{~min})$ of the $\mathrm{OO}$ MEP (Fig. 6A, top panel), whereas ARM + INB resulted in only a transient increase (bottom panel). Similarly, the group data revealed a long-lasting (>60 min) increase of OO MEP sum with FACE + INB (Fig. 6B, top panel, filled squares) but no change with ARM + INB (Fig. 6B, top panel, open circles). Conversely, the BB MEP sum increased only transiently with FACE + INB (Fig. 6B, bottom panel, filled squares), but a long-lasting increase occurred with ARM + INB (Fig. 6B, bottom panel, open circles). This inverse behavior of changes in MEP sum in the OO and BB muscles with experimental manipulation was reflected in a trend toward a significant interaction between muscle and manipulation $(F=8.17 ; p=0.065)$ in the repeated-measures three-way ANOVA. The apparently more sustained increase of OO MEP sum with FACE + INB compared with the increase of BB MEP
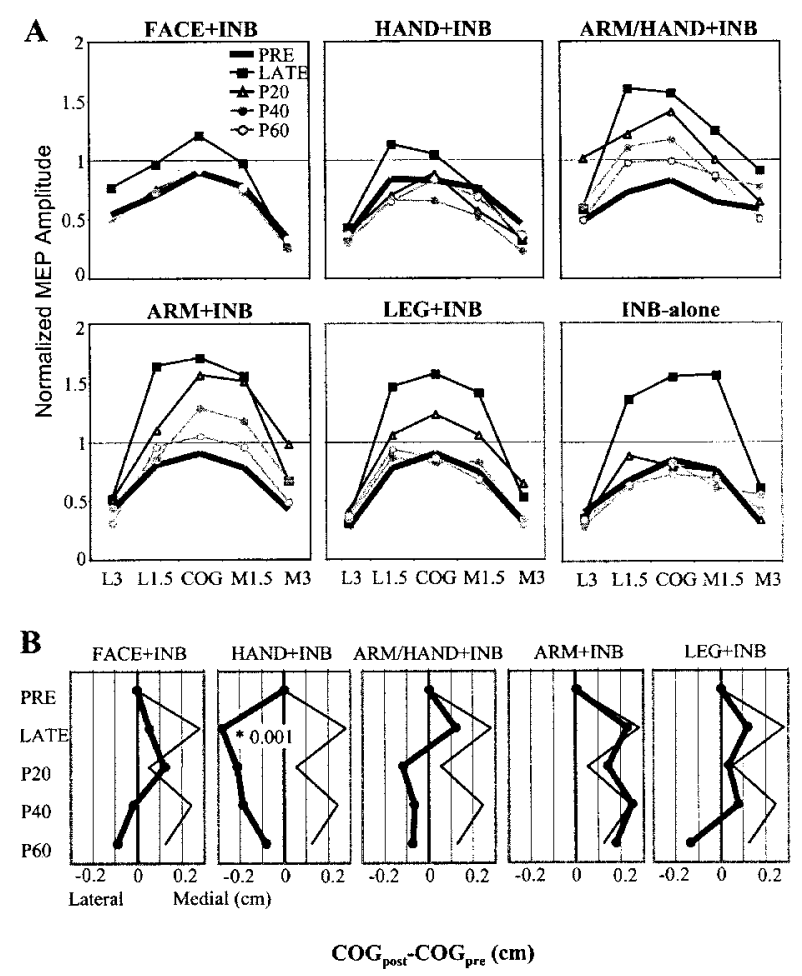

Figure 5. A, MEP mapping of the biceps muscle. MEPs were elicited by focal TMS at the COG of the biceps map and at sites 1.5 and $3 \mathrm{~cm}$ anterior-lateral (L1.5, L3) and posterior-medial (M1.5, M3) from COG. The different curves refer to the different times of measurement, as indicated in the inset (for abbreviations, see Fig. 1). MEP amplitudes are normalized to the individual maximum MEP during PRE (usually, but not always obtained at $\mathrm{COG}$ ). The different manipulations are indicated at the top of each diagram (for abbreviations, see Fig. 1). $B$, Changes of the COG along the lateral-to-medial axis. Data are from the MEP maps in $A$. The thin curve in each diagram refers to the INB-alone (control) condition, and thick curves refer to the experimental manipulation shown at the top of each diagram (for abbreviations, see Fig. 1). The asterisk indicates a significant lateral shift of the COG with HAND + INB compared with INB-alone.

sum with ARM + INB (Fig. 6B) was not significant. Finally, the shifts of the COG also showed an inverse pattern with experimental manipulation for the $\mathrm{OO}$ and $\mathrm{BB}$ muscles (Fig. 6C). These effects, however, were not significant (muscle $\times$ manipulation interaction: $F=4.34 ; p=0.13$ ).

These data suggest that the interaction between the face and arm representations in the experimentally disinhibited human motor cortex is mutually inhibitory. This extends the above findings that had revealed such an inhibitory interaction only for the direction from the face to the arm representation.

\section{DISCUSSION}

\section{Within-representation plasticity}

We have shown previously that the ischemic nerve block of the hand (the INB-alone control condition) results in a rapid decrease of the content of GABA, the main inhibitory neurotransmitter, in the deprived sensorimotor cortex (Levy et al., 1999). The associated release from cortical inhibition results in a transient increase of motor output from the arm and hand representations (Brasil-Neto et al., 1992; Ziemann et al., 1998a; McNulty et al., 2002) and is permissive for the expression of long-lasting stimulation-induced (Ziemann et al., 1998a,b) and use-dependent plasticity (Ziemann et al., 2001). 

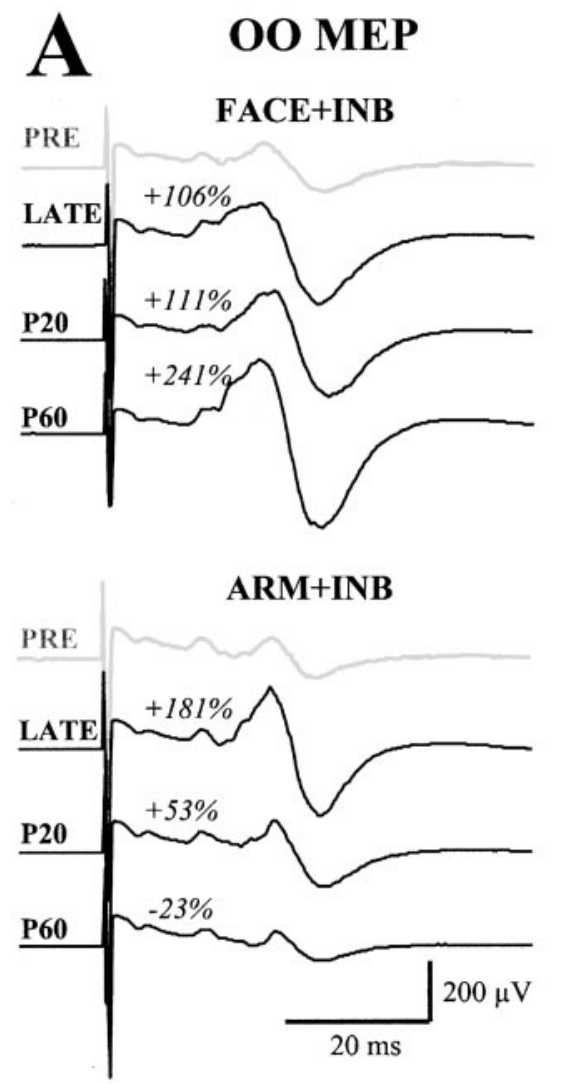

B
OO MEP sum

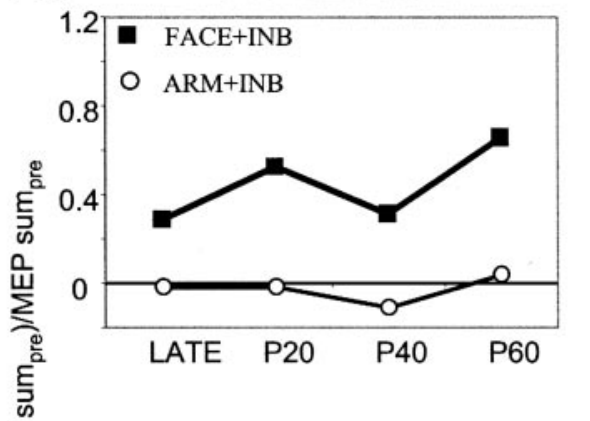

BB MEP sum

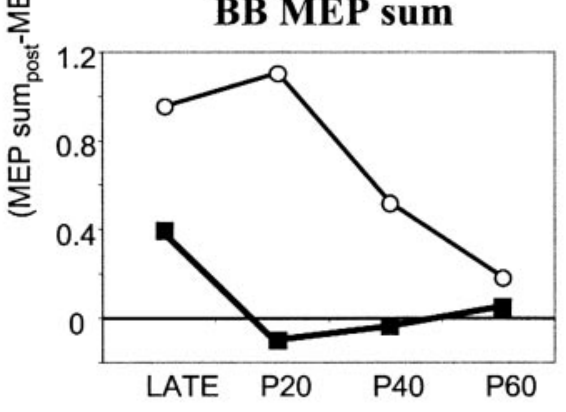

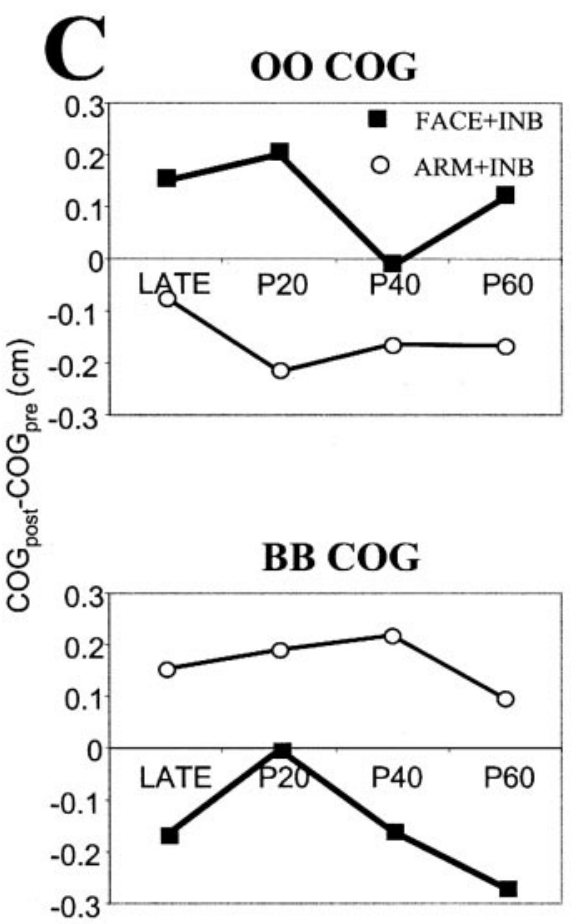

Figure 6. A, MEPs in the OO muscle of the same subject as in Figure 3, measured before (gray curve, PRE), LATE into, and 20 and 60 min after (P20, P60) FACE + INB (top panel) or ARM + INB (bottom panel, for abbreviations see Fig. 1). All MEPs were elicited $6 \mathrm{~cm}$ lateral from the COG of the biceps $(B B)$, and are averages of five trials. Numbers indicate the percentage change of MEP amplitude compared with the MEP measured during PRE. Note that ARM + INB resulted in an only transient increase of the OO MEP, whereas the increase with FACE + INB was long-lasting. $B$, Group data $(n=4)$ of changes in OO (top panel) and BB (bottom panel) MEP sum induced by two different experimental manipulations, FACE + INB ( filled squares) and ARM + INB (open circles). Note that, similar to the single subject data in Figure $6 \mathrm{~A}$, FACE + INB resulted in a long-lasting increase of MEP sum in the OO but not BB. Conversely, ARM + INB led to a long-lasting increase of the MEP sum in BB but not OO. $C$, Group data $(n=4)$ of changes in the COG of the $\mathrm{OO}$ and $\mathrm{BB}$ maps induced by two experimental manipulations. Positive values ( $y$-axis, in centimeters) denote shifts of the COG in the medial direction, and negative values shifts in the lateral direction. Otherwise, same arrangement and conventions as in $B$.

The present experiments show, for the first time, that the long-lasting (>60 min) stimulation-induced increase of motor output from the arm representation is input-specific because it required stimulation of that representation (ARM + INB and ARM/HAND + INB conditions), whereas it did not occur with stimulation of nearby representations (FACE + INB, HAND + INB, and LEG + INB conditions). Therefore, input specificity, in addition to cooperatively that describes a threshold phenomenon for induction (Ziemann et al., 1998a) and NMDA receptor dependence (Ziemann et al., 1998b) characterizes this form of stimulation-induced within-representation plasticity in human motor cortex. These three properties also characterize long-term potentiation (LTP) (Bliss and Collingridge, 1993). This raises the hypothesis that similar mechanisms underlie this form of plasticity and LTP.

\section{Across-representation plasticity between the hand and the arm representation}

Stimulation of the hand representation (the HAND + INB condition) canceled the transient increase of motor output from the arm representation obtained with the INB-alone condition and even led to a long-lasting decrease of motor output from the arm representation (Fig. 4). This suggests an inhibitory action from the hand representation to the arm representation. This interaction could be mediated by the extensive horizontal intrinsic connectivity within the arm/hand representation in motor cortex (Sanes and Donoghue, 2000). Intracortical microstimulation in monkey motor cortex resulted in local facilitation of neurons close to the stimulation electrode but principally inhibition of distant neurons in the surround (Baker et al., 1998). Paired-pulse TMS experiments in humans also showed that intracortical facilitation is local (Ziemann et al., 1996), whereas inhibition within motor cortex is obtained over larger distances, even between different motor representations (Kujirai et al., 1993). If such a local-facilitation/surround-inhibition model applied to the present experiments, then the long-lasting decrease of motor output from the arm representation by stimulation of the laterally adjacent and partly overlapping hand representation may be explained through potentiation of inhibitory connections between the hand and arm representations, similar to LTP of inhibitory inputs in rat visual cortex (Komatsu, 1994). The lateral shift of the BB COG (Fig. 5) supports this local-facilitation/surroundinhibition model. Facilitation would occur locally beneath the stimulation site, enhancing the most laterally located parts of the arm representation, whereas surround inhibition would occur in some distance from the stimulation site, suppressing the main more medially located part of the arm representation. 


\section{Across-representation plasticity between the face and the arm representation}

Stimulation of the face representation (the FACE + INB condition) canceled the transient increase of motor output from the arm representation obtained in the INB-alone condition (control), but did not result in the long-lasting decrease observed with the HAND + INB condition (Fig. 4). Furthermore, the changes of the face and arm representations showed an inverse and mutually inhibitory behavior with the FACE + INB and ARM + INB conditions (Fig. 6B). The mechanisms of these interactions are unclear. In humans, the face and arm/hand representations are topographically distinct (Grafton et al., 1991; Classen et al., 1998). Furthermore, intracortical microstimulation mapping in nonhuman primate motor cortex even showed a zone of unresponsive motor cortex that separated the face and forelimb representations (Waters et al., 1990). In humans, direct cortex stimulation of the face representation did not result in excitability changes of the hand representation (Ashby et al., 1999). Finally, although there exist limited horizontal corticocortical connections between the vibrissae and forelimb representations in rat motor cortex (Huntley, 1997), no such direct corticocortical connections were found between the face and arm/hand representations in nonhuman primate motor cortex (Huntley and Jones, 1991). Therefore, indirect connectivity must be invoked to explain the present findings and those of others. A competitive activity-dependent interaction between the face and arm/hand representations of motor cortex was demonstrated in upper limb amputees. The interaction is implied by a medial shift of the face representation (Pascual-Leone et al., 1996; Karl et al., 2001) and a lateral shift of the arm representation (Pascual-Leone et al., 1996) toward the deprived hand representation. Patients with facial palsy showed an enlargement and lateral extension of the hand representation toward the face representation (Rijntjes et al., 1997). Conversely, patients with hemifacial spasm had a reduced size of the hand representation that was reversed after successful treatment with botulinum toxin (Liepert et al., 1999). The inhibitory effects between the face and arm representations in the present experiments are explained best by mechanisms similar to the ones discussed above for the interaction between hand and arm representation. The shorter duration of these effects in the FACE + INB compared with the HAND + INB condition is in agreement with the less direct connectivity or larger anatomical distance between the face and arm representations.

The inhibitory effects from the face to the arm representation were borne out only during release from cortical inhibition, whereas stimulation of the face in the absence of INB (the FACE-alone condition) did not produce any effects on the arm representation (Fig. 4). This is consistent with the permissive effects of local disinhibition on the expression of stimulationinduced plasticity in rat motor cortex (Hess and Donoghue, 1994; Hess et al., 1996). It appears that the motor cortex has a higher threshold for stimulation-induced plasticity than other areas of the neocortex (Castro-Alamancos et al., 1995; Castro-Alamancos and Connors, 1996). The reasons for this difference are incompletely understood, but may include differences in cytoarchitecture (missing layer IV in motor cortex) and in NMDA receptor subtypes (Castro-Alamancos and Connors, 1996).

\section{Across-representation plasticity between the leg and the arm representation}

Stimulation of the leg representation (the LEG + INB condition) did not significantly alter the transient increase of motor output from the arm representation obtained in the INB-alone condition (Fig. 4). This contrasts clearly with the inhibitory effects obtained with the FACE + INB condition (see above). The difference cannot be explained by greater anatomical distance from the BB COG $(3.7 \pm 0.7 \mathrm{~cm}$ for TA, $5.0 \pm 0.3 \mathrm{~cm}$ for OO). The lack of effects may point to a lack of inhibitory interactions from the leg to the arm representation. This is unlikely because a previous TMS study found an inhibitory effect on MEP size of a hand muscle by conditioning stimulation over the leg area (Kujirai et al., 1993). More likely, the lack of an interaction from the leg to the arm representation is caused by the fact that the leg representation is not adjacent to the deafferented hand cortex but separated by the arm and trunk representations. Consistent with this idea, reshaping in monkey motor cortex (Schieber and Deuel, 1997) and somatosensory cortex (Pons et al., 1991; Florence and Kaas, 1995) occurred only for those representations directly adjacent to the deprived cortex.

\section{Functional relevance of the present findings}

The human primary motor cortex is no longer considered as simply the final common pathway for motor task execution but also as a dynamically reshaped site for acquisition and consolidation of motor skills (Pascual-Leone et al., 1994; Karni et al., 1995; Honda et al., 1998; Muellbacher et al., 2001; Muellbacher et al., 2002). The present findings of stimulation-induced withinrepresentation increase versus across-representation decrease of motor output from representations adjacent to the deprived cortex suggest that this reshaping is an activity-driven, potentially bidirectional, and competitive process. Within-representation plasticity may operate after incomplete brain lesions when repeated training leads to increased motor output from the affected representation with gain of motor function (Liepert et al., 1998). Across-representation plasticity, on the other hand, may operate when a body part representation is more substantially damaged, and the disinhibited perilesional region, with a lower threshold for LTP induction than "normal" cortex (Hagemann et al., 1998), may serve as a substrate for motor recovery (Nudo and Milliken, 1996; Nudo et al., 1996). In different settings, these plastic changes can play a maladaptive role. For example, upper limb amputees showed an extensive medial shift of the face representation in motor cortex toward the deprived hand cortex, associated with severe phantom limb pain, unless the stump was extensively "trained" by use of a myoelectric prosthesis (Lotze et al., 1999).

In summary, manipulation of within- and across-representation plasticity in human cortex may have the potential for a broad use in rehabilitation to enhance plasticity when it plays an adaptive role and to ward it off when it is maladaptive.

\section{REFERENCES}

Ashby P, Reynolds C, Wennberg R, Lozano AM, Rothwell J (1999) On the focal nature of inhibition and facilitation in the human motor cortex. Clin Neurophysiol 110:550-555.

Baker SN, Oliver E, Lemon R (1998) An investigation of the intrinsic circuitry of the motor cortex of the monkey using intra-cortical microstimulation. Exp Brain Res 123:397-411.

Bliss TV, Collingridge GL (1993) A synaptic model of memory: longterm potentiation in the hippocampus. Nature 361:31-39.

Brasil-Neto JP, Cohen LG, Pascual-Leone A, Jabir F K, Wall RT, Hallett M (1992) Rapid reversible modulation of human motor outputs after transient deafferentation of the forearm: a study with transcranial magnetic stimulation. Neurology 42:1302-1306.

Castro-Alamancos MA, Connors BW (1996) Short-term synaptic enhancement and long-term potentiation in neocortex. Proc Natl Acad Sci USA 93:1335-1339.

Castro-Alamancos MA, Donoghue JP, Connors BW (1995) Different 
forms of synaptic plasticity in somatosensory and motor areas of the neocortex. J Neurosci 15:5324-5333.

Chen R, Classen J, Gerloff C, Celnik P, Wassermann EM, Hallett M, Cohen LG (1997) Depression of motor cortex excitability by lowfrequency transcranial magnetic stimulation. Neurology 48:1398-1403.

Cheyne D, Kristeva R, Deecke L (1991) Homuncular organization of human motor cortex as indicated by neuromagnetic recordings. Neurosci Lett 122:17-20.

Classen J, Knorr U, Werhahn KJ, Schlaug G, Kunesch E, Cohen LG, Seitz RJ, Benecke R (1998) Multimodal output mapping of human central motor representation on different spatial scales. J Physiol (Lond) 512:163-179.

Donoghue JP, Suner S, Sanes JN (1990) Dynamic organization of primary motor cortex output to target muscles in adult rats. II. Rapid reorganization following motor nerve lesions. Exp Brain Res 79:492-503.

Florence SL, Kaas JH (1995) Large-scale reorganization at multiple levels of the somatosensory pathway follows therapeutic amputation of the hand in monkeys. J Neurosci 15:8083-8095.

Gracely RH, McGrath F, Dubner R (1978) Ratio scales of sensory and affective verbal pain descriptors. Pain 5:5-18.

Grafton ST, Woods RP, Mazziotta JC, Phelps ME (1991) Somatotopic mapping of the primary motor cortex in humans: activation studies with cerebral blood flow and positron emission tomography. J Neurophysiol 66:735-743.

Graham Brown T, Sherrington CS (1912) On the stability of a cortical point. Proc R Soc Lond B Biol Sci 85:250-277.

Grünbaum ASF, Sherrington CS (1903) Observations on the physiology of the cerebral cortex of the anthropoid apes. Proc R Soc Lond B Biol Sci 72:152-155.

Hagemann G, Redecker C, Neumann-Haefelin T, Freund H-J, Witte O (1998) Increased long-term potentiation in the surround of experimentally induced focal cortical infarction. Ann Neurol 44:255-258.

Hess G, Donoghue JP (1994) Long-term potentiation of horizontal connections provides a mechanism to reorganize cortical motor maps. J Neurophysiol 71:2543-2547.

Hess G, Aizenman CD, Donoghue JP (1996) Conditions for the induction of long-term potentiation in layer II/III horizontal connections of the rat motor cortex. J Neurophysiol 75:1765-1778.

Honda M, Deiber MP, Ibanez V, Pascual-Leone A, Zhuang P, Hallett M (1998) Dynamic cortical involvement in implicit and explicit motor sequence learning. A PET study. Brain 121:2159-2173.

Huntley GW (1997) Correlation between patterns of horizontal connectivity and the extent of short-term representational plasticity in rat motor cortex. Cereb Cortex 7:143-156.

Huntley GW, Jones EG (1991) Relationship of intrinsic connections to forelimb movement representations in monkey motor cortex: a correlative anatomical and physiological study. J Neurophysiol 66:390-413.

Kaas JH (1991) Plasticity of sensory and motor maps in adult mammals. Annu Rev Neurosci 14:137-167.

Kaneko K, Kawai S, Fuchigami Y, Morita H, Ofuji A (1996) The effect of current direction induced by transcranial magnetic stimulation on the corticospinal excitability in human brain. Electroencephalogr Clin Neurophysiol 101:478-482.

Karl A, Birbaumer N, Lutzenberger W, Cohen LG, Flor H (2001) Reorganization of motor and somatosensory cortex in upper extremity amputees with phantom limb pain. J Neurosci 21:3609-3618.

Karni A, Meyer G, Jezzard P, Adams MM, Turner R, Ungerleider LG (1995) Functional MRI evidence for adult motor cortex plasticity during motor skill learning. Nature 377:155-158.

Komatsu Y (1994) Age-dependent long-term potentiation of inhibitory synaptic transmission in rat visual cortex. J Neurosci 14:6488-6499.

Kujirai T, Caramia MD, Rothwell JC, Day BL, Thompson PD, Ferbert A, Wroe S, Asselman P, Marsden CD (1993) Corticocortical inhibition in human motor cortex. J Physiol (Lond) 471:501-519.

Levy LM, Ziemann U, Chen R, Cohen LG (1999) Rapid modulation of GABA in human cortical plasticity demonstrated by magnetic resonance spectroscopy. Neurology 52:A 88 .

Liepert J, Miltner WHR, Bauder H, Sommer M, Dettmers C, Weiller C (1998) Motor cortex plasticity during constraint-induced movement therapy in stroke patients. Neurosci Lett 250:5-8.

Liepert J, Oreja-Guevara C, Cohen LG, Tegenthoff M, Hallett M, Malin J-P (1999) Plasticity of cortical hand muscle representation in patients with hemifacial spasm. Neurosci Lett 272:33-36.

Lotze M, Grodd W, Birbaumer N, Erb M, Huse E, Flor H (1999) Does use of myoelectric prosthesis prevent cortical reorganization and phantom limb pain? Nat Neurosci 2:501-502.
Max MB, Lynch SA, Muir J, Shoaf SE, Smoller B, Dubner R (1992) Effects of desipramine, amitriptyline, and fluoxetine on pain in diabetic neuropathy. N Engl J Med 326:1250-1256.

McNulty PA, Macefield VG, Taylor JL, Hallett M (2002) Cortically evoked neural volleys to the human hand are increased during ischaemic block of the forearm. J Physiol (Lond) 538:279-288.

Muellbacher W, Ziemann U, Boroojerdi B, Cohen LG, Hallett M (2001) Role of the human motor cortex in rapid motor learning. Exp Brain Res 136:431-438.

Muellbacher W, Ziemann U, Wissel J, Dang N, Kofler M, Facchini S, Boroojerdi B, Poewe W, Hallett M (2002) Early consolidation in human primary motor cortex. Nature 415:640-644.

Nudo RJ, Jenkins WM, Merzenich MM (1990) Repetitive microstimulation alters the cortical representation of movements in adult rats. Somatosens Mot Res 7:463-483.

Nudo RJ, Milliken GW (1996) Reorganization of movement representations in primary motor cortex following focal ischemic infarcts in adult squirrel monkeys. J Neurophysiol 75:2144-2149.

Nudo RJ, Wise BM, SiFuentes F, Milliken GW (1996) Neural substrates for the effects of rehabilitative training on motor recovery after ischemic infarct. Science 272:1791-1794.

Pascual-Leone A, Grafman J, Hallett M (1994) Modulation of cortical motor output maps during development of implicit and explicit knowledge. Science 263:1287-1289.

Pascual-Leone A, Peris M, Tormos JM, Pascual AP, Catala MD (1996) Reorganization of human cortical motor output maps following traumatic forearm amputation. NeuroReport 7:2068-2070.

Penfield W, Boldrey E (1937) Somatic motor and sensory representation in the cerebral cortex of man as studied by electrical stimulation. Brain 60:389-443.

Pons TP, Garraghty PE, Ommaya AK, Kaas JH, Taub E, Mishkin M (1991) Massive cortical reorganization after sensory deafferentation in adult macaques. Science 252:1857-1860.

Rao SM, Binder JR, Hammeke TA, Bandettini PA, Bobholz JA, Frost JA, Myklebust BM, Jacobson RD, Hyde JS (1995) Somatotopic mapping of the human primary motor cortex with functional magnetic resonance imaging. Neurology 45:919-924.

Recanzone GH, Merzenich MM, Dinse HR (1992) Expansion of the cortical representation of a specific skin field in primary somatosensory cortex by intracortical microstimulation. Cereb Cortex 2:181-196.

Rijntjes M, Tegenthoff M, Liepert J, Leonhardt G, Kotterba S, Muller S, Kiebel S, Malin JP, Diener HC, Weiller C (1997) Cortical reorganization in patients with facial palsy. Ann Neurol 41:621-630.

Rossini PM, Barker AT, Berardelli A, Caramia MD, Caruso G, Cracco RQ, Dimitrijevic MR, Hallett M, Katayama Y, Lücking CH, Maertens de Noordhout AL, Marsden CD, Murray NMF, Rothwell JC, Swash M, Tomberg C (1994) Non-invasive electrical and magnetic stimulation of the brain, spinal cord and roots: basic principles and procedures for routine clinical application. Report of an IFCN committee. Electroencephalogr Clin Neurophysiol 91:79-92.

Sanes JN, Donoghue JP (2000) Plasticity and primary motor cortex. Annu Rev Neurosci 23:393-415.

Sanes JN, Suner S, Lando JF, Donoghue JP (1988) Rapid reorganization of adult rat motor cortex somatic representation patterns after motor nerve injury. Proc Natl Acad Sci USA 85:2003-2007.

Schieber MH, Deuel RK (1997) Primary motor cortex reorganization in a long-term monkey amputee. Somatosens Mot Res 14:157-167.

Wassermann EM, McShane LM, Hallett M, Cohen LG (1992) Noninvasive mapping of muscle representations in human motor cortex. Electroencephalogr Clin Neurophysiol 85:1-8.

Waters RS, Samulack DD, Dykes RW, McKinley PA (1990) Topographic organization of baboon primary motor cortex: face, hand forelimb, and shoulder representation. Somatosens Mot Res 7:485-514.

Ziemann U, Rothwell JC, Ridding MC (1996) Interaction between intracortical inhibition and facilitation in human motor cortex. J Physiol (Lond) 496:873-881.

Ziemann U, Corwell B, Cohen LG (1998a) Modulation of plasticity in human motor cortex after forearm ischemic nerve block. J Neurosci 18:1115-1123.

Ziemann U, Hallett M, Cohen LG (1998b) Mechanisms of deafferentation-induced plasticity in human motor cortex. J Neurosci 18:7000-7007.

Ziemann U, Muellbacher W, Hallett M, Cohen LG (2001) Modulation of practice-dependent plasticity in human motor cortex. Brain 124: 1171-1181. 